\title{
On a method for solving nonlinear integro differential equa- tion of order $n$
}

\author{
M. A. Abdou ${ }^{a}$, M. I. Youssef ${ }^{b, a, *}$ \\ ${ }^{a}$ Department of Mathematics, Faculty of Education, Alexandria University, Alexandria, 21526, Egypt. \\ ${ }^{b}$ Department of Mathematics, College of Science, Jouf University, P. O. Box 2014, Sakaka, Saudi Arabia.
}

\begin{abstract}
This work is concerned with the study of a general class of nonlinear integro-differential equations of order $\mathrm{n}$. Using a suitable transformation, we derive an equivalent nonlinear Fredholm-Volterra integral equation (NF-VIE) to this class of equations. The existence of continuous solutions for the NF-VIE is investigated subject to the verification of some sufficient conditions. We apply the modified Adomian's decomposition method (MADM) and homotopy analysis method (HAM) to solve this NF-VIE. The convergence and error estimate of the approximate solution are also studied. The numerical results in this article show that the HAM technique may give an approximate solution with high accuracy and convergence rate faster than the one obtained using the MADM technique provided the convergence control parameter $\hbar$ is properly chosen when applying the HAM.
\end{abstract}

Keywords: Integro-differential equations, existence, uniqueness, modified Adomian's decomposition method, homotopy analysis method.

2020 MSC: 45G99, 34A12, 45L05, 45G10.

(C)2022 All rights reserved.

\section{Introduction}

Many applicable models in physical, biological and chemical sciences can be described successfully using integro-differential equations. For example, the biological population models rely on the delayed Volterra integro-differential equations, systems of integro-differential equations characterize the evolution of nuclear reactor in a continuous medium, and many other problems in visco-elasticity, mechanics and economics as well. Furthermore, converting initial and boundary value problems yields these types of equations. In operator theory, integral and differential operators represent the main source for supporting theories through a lot of applicable examples. So, investigating this class of equations is of great significance. The majority of non-linear equations do not have a closed form analytical solution. Consequently, many techniques such as the perturbation methods, which are commonly based on transferring the nonlinear problem to infinite number of linear sub problems through the perturbation parameters, are introduced to get approximate solutions for these problems in different fields of sciences (see $[17,26,27]$

\footnotetext{
*Corresponding author

Email addresses: abdella_777@yahoo.com (M. A. Abdou), miyoussef283@gmail .com (M. I. Youssef)

doi: $10.22436 /$ jmcs.025.04.03
} 
and the cited references therein). Also, there are non perturbation methods such as the Adomian decomposition method (ADM). After George Adomian presented his method in the 1980s [1-3], it has become one of the remarkable techniques used to find exact and approximate solutions for a wide class of linear as well as non-linear integral equations and many other types of equations as well. In addition, this method is providing us a numerical algorithm based on applying the so called Adomian's polynomial to solve nonlinear equations. For example, Abdou et al. [7] used the ADM to solve a nonlocal stochastic fractional integro-differential equation and proved the mean square convergence of the infinite series solution when the problem has a unique solution. Also, the authors estimated the mean square error between the truncated series and exact solution. Xie [31] solved Volterra integral equation of the second kind using the ADM after proposing a new modification on it. Singh et al. [29] obtained approximate solution of the Urysohn integral equation using the ADM. Issa et al. [18] applied the MADM to solve first order integro-differential equation of the fuzzy-Volterra type numerically and many researchers utilized the $\mathrm{ADM}$ and MADM for solving nonlinear equations as well, (see for more explanations $[5,6,8-12,14,25]$ and the references mentioned therein). Another elegant semi-analytical method is called the homotopy analysis method (HAM). This technique is considered one of the most used methods for solving linear and nonlinear equations. This algorithm is based on the concept of homotopy from topology and was first introduced in 1992 by Liao in [20-22]. Since then, many researchers compared this method with many techniques already used before and also, used it to solve many challenging problems in science. For instance, Kurt et al. [19] found an approximate solution to the modified Burgers equation using the HAM. Singh et al. [28] applied the HAM combined with the Sumudu transform method to solve the Drinfeld-Sokolov-Wilson equation of fractional type. Maitama et al. [24] applied the HAM and local fractional calculus to find solution for some non differentiable problems on the Contor sets. In addition, Liao et al. have proved that the ADM is a special case of the HAM when the control parameter $\hbar=-1$ in the homotopy operator, see $[9,13,16,21,23]$ for more explanations about the HAM.

In this work, we investigate the analytical and numerical solutions for a general class of integrodifferential equations of order $n$ on the form

$$
\begin{aligned}
\mu \phi^{(n)}(x)+B_{1}(x) & \phi^{(n-1)}(x)+B_{2}(x) \phi^{(n-2)}(x)+\cdots+B_{n}(x) \phi(x) \\
& =f(x)-\lambda \int_{a}^{b} \psi(x, y)[\phi(y)]^{p} d y, a \leqslant x \leqslant b, 0<p \leqslant n<\infty,
\end{aligned}
$$

subject to the initial conditions

$$
\phi^{(k)}(a)=q_{k}, q_{k}<\infty, \forall 0 \leqslant k \leqslant n-1,
$$

where $\phi^{(n)}(x)=\frac{d^{n} \phi}{d x^{n}}, n<\infty$. The functions $f, \psi$ and $B_{i}, 1 \leqslant i \leqslant n$, are known while $\phi$ is the sought function. The parameters $\{\mu, \lambda\} \in \mathbb{R} \backslash\{0\}$ and $\lambda$ may has a physical meaning. We prove the existence of solutions for Eq. (1.1) under the condition (1.2) (see Def. (2.2)) and derive the necessary conditions which make the solution unique. We employ the MADM to find the series solution for Eq. (1.1) under (1.2). Furthermore, we prove the convergence of this series to the exact solution (when it exists) and estimate the truncation error using this method. The recursive equation based on applying the HAM to solve Eq. (1.1) under (1.2) is given as well. We believe that the current form of Eq. (1.1) is not discussed before. This work shows that applying the HAM can give results better than the MADM in solving the proposed problem provided the convergence control parameter $\hbar$ is properly chosen when applying the HAM. The rest of this paper is ordered as the following. In the "Existence and uniqueness results" section we prove the existence of at least one continuous solution to the proposed model and state with proof the sufficient conditions which make the solution unique. The MADM and HAM are applied in sections "The Modified Adomian's decomposition method" and "The homotopy analysis method", respectively. Next, the analytical and numerical results are introduced in section "Analytical and numerical results" to make clear understanding of applying the above two techniques in solving the present problem. Finally, a conclusion and discussion are given in section "Conclusion". 


\section{Existence and uniqueness results}

In what follows, we assume the following hypotheses:

(i) the maps $x \longmapsto B_{i}(x)$ are elements in the space $C([a, b], \mathbb{R}), \forall 1 \leqslant i \leqslant n$;

(ii) the map $x \longmapsto f(x)$ belongs to the space $C^{n}([a, b], \mathbb{R})$;

(iii) the kernel $\psi(x, y)$ of the integral term is known and has a weak singular part in general at $x=y$.

Theorem 2.1. Suppose the hypotheses (i)-(iii) are verified. Then the integro-differential equation (1.1) subject to the initial conditions (1.2) is equivalent to the following NF-VIE:

$$
\mu g(x)+\int_{a}^{x} \Phi(x, t) g(t) d t=F(x)-\lambda \int_{a}^{b} \sum_{l=0}^{p} M(x, y ; l)\left[\int_{a}^{y}(y-t)^{n-1} g(t) d t\right]^{l} d y,
$$

where

$$
\begin{aligned}
& g(x):=\phi^{(n)}(x), \quad \Phi(x, t):=\sum_{j=1}^{n} \frac{(x-t)^{j-1}}{(j-1) !} B_{j}(x), \\
& F(x):=f(x)-L(x), \quad M(x, y ; l):=\frac{p ! \psi(x, y)}{l !(p-l) ![(n-1) !]^{l}}\left(\sum_{j=0}^{n-1} \frac{(y-a)^{j}}{j !} q_{j}\right)^{p-l},
\end{aligned}
$$

and the function $\mathrm{L}(\mathrm{x})$ is given by the formula

$$
L(x):=\sum_{i=0}^{n-1}\left[\sum_{j=i}^{n-1} \frac{(x-a)^{j-i}}{(j-i) !} q_{j} B_{n-i}(x)\right] .
$$

Proof. Let $\phi^{(n)}(x)=g(x)$, where the map $x \longmapsto g(x)$ is a real valued continuous function defined on $[a, b]$. Integrating both sides $n$-times from 0 to $x$ and then using initial conditions (1.2) give

$$
\phi(x)=\sum_{j=0}^{n-1} \frac{(x-a)^{j}}{j !} q_{j}+\int_{a}^{x} \frac{(x-t)^{n-1}}{(n-1) !} g(t) d t .
$$

Furthermore, it is easy to see that

$$
\begin{aligned}
{[\phi(x)]^{p} } & =\left[\sum_{j=0}^{n-1} \frac{(x-a)^{j}}{j !} q_{j}+\int_{a}^{x} \frac{(x-t)^{n-1}}{(n-1) !} g(t) d t\right]^{p} \\
& =\sum_{l=0}^{p} \frac{p !}{l !(p-l) !}\left(\sum_{j=0}^{n-1} \frac{(x-a)^{j}}{j !} q_{j}\right)^{p-l}\left(\int_{a}^{x} \frac{(x-t)^{n-1}}{(n-1) !} g(t) d t\right)^{l} .
\end{aligned}
$$

Using the above results to substitute in Eq. (1.1) yields

$$
\begin{aligned}
& \mu g(x)+\int_{a}^{x} \sum_{j=1}^{n} \frac{(x-t)^{j-1}}{(j-1) !} B_{j}(x) g(t) d t+\left[q_{n-1} B_{1}(x)+\left[q_{n-2}+(x-a) q_{n-1}\right] B_{2}(x)+\cdots\right. \\
& \left.+\sum_{j=2}^{n-1} \frac{(x-a)^{j-2}}{(j-2) !} q_{j} B_{n-2}(x)+\sum_{j=1}^{n-1} \frac{(x-a)^{j-1}}{(j-1) !} q_{j} B_{n-1}(x)+\sum_{j=0}^{n-1} \frac{(x-a)^{j}}{j !} q_{j} B_{n}(x)\right] \\
& =f(x)-\lambda \int_{a}^{b} \psi(x, y) \sum_{l=0}^{p} \frac{p !}{l !(p-l) ![(n-1) !]}\left(\sum_{j=0}^{n-1} \frac{(y-a)^{j}}{j !} q_{j}\right)^{p-l}\left(\int_{a}^{y}(y-t)^{n-1} g(t) d t\right)^{l} d y .
\end{aligned}
$$


But

$$
\begin{aligned}
\sum_{i=0}^{n-1} & {\left[\sum_{j=i}^{n-1} \frac{(x-a)^{j-i}}{(j-i) !} q_{j} B_{n-i}(x)\right]=\left[\sum_{j=0}^{n-1} \frac{(x-a)^{j}}{j !} q_{j} B_{n}(x)+\sum_{j=1}^{n-1} \frac{(x-a)^{j-1}}{(j-1) !} q_{j} B_{n-1}(x)\right.} \\
& \left.+\sum_{j=2}^{n-1} \frac{(x-a)^{j-2}}{(j-2) !} q_{j} B_{n-2}(x)+\cdots+\left[q_{n-2}+(x-a) q_{n-1}\right] B_{2}(x)+q_{n-1} B_{1}(x)\right] .
\end{aligned}
$$

Substituting Eq. (2.6) in Eq. (2.5) we have

$$
\begin{aligned}
& \mu g(x)+\int_{a}^{x} \sum_{j=1}^{n} \frac{(x-t)^{j-1}}{(j-1) !} B_{j}(x) g(t) d t \\
& =f(x)-\sum_{i=0}^{n-1}\left[\sum_{j=i}^{n-1} \frac{(x-a)^{j-i}}{(j-i) !} q_{j} B_{n-i}(x)\right] \\
& \quad-\lambda \int_{a}^{b} \psi(x, y) \sum_{l=0}^{p} \frac{p !}{l !(p-l) ![(n-1) !]^{l}}\left(\sum_{j=0}^{n-1} \frac{(y-a)^{j}}{j !} q_{j}\right)^{p-l}\left(\int_{a}^{y}(y-t)^{n-1} g(t) d t\right)^{l} d y .
\end{aligned}
$$

Set

$$
\begin{aligned}
\Phi(x, t) & :=\sum_{j=1}^{n} \frac{(x-t)^{j-1}}{(j-1) !} B_{j}(x) \\
M(x, y ; l) & :=\frac{p ! \psi(x, y)}{l !(p-l) ![(n-1) !]^{l}}\left(\sum_{j=0}^{n-1} \frac{(y-a)^{j}}{j !} q_{j}\right)^{p-l}, \\
L(x) & :=\sum_{i=0}^{n-1}\left[\sum_{j=i}^{n-1} \frac{(x-a)^{j-i}}{(j-i) !} q_{j} B_{n-i}(x)\right]
\end{aligned}
$$

Then, substituting in Eq. (2.5) gives

$$
\mu g(x)+\int_{a}^{x} \Phi(x, t) g(t) d t=F(x)-\lambda \int_{a}^{b} \sum_{l=0}^{p} M(x, y ; l)\left[\int_{a}^{y}(y-t)^{n-1} g(t) d t\right]^{l} d y
$$

where

$$
F(x):=f(x)-L(x)
$$

Conversely, substituting $g(x)=\phi^{(n)}(x)$ in Eq. (2.1) and then using integration by parts will give Eq. (1.1) after simplifying it through utilizing Eqs. (2.2), (2.3), and (1.2). This completes the proof.

Definition 2.2. The solution for the integro-differential Eq. (1.1) means obtaining the function $\phi \in$ $\mathrm{C}^{\mathrm{n}}([\mathrm{a}, \mathrm{b}] ; \mathbb{R})$ which satisfies Eq. (1.1), and initial conditions (1.2).

Now, we suppose the following hypothesis.

(iv) The given kernel $\psi(x, y)$ is continuous, $\forall(x, y) \in[a, b]^{2}$.

Theorem 2.3. Assume the conditions (i), (ii), and (iv) are satisfied. Then the NF-VIE (2.1) has at least one continuous solution. 
Proof. Let $\mathrm{W}$ be an integral operator defined by Eq. (2.8):

$$
W(g)(x)=\frac{1}{\mu} F(x)-\frac{1}{\mu} \int_{a}^{x} \Phi(x, t) g(t) d t-\frac{\lambda}{\mu} \int_{a}^{b} \sum_{l=0}^{p} M(x, y ; l)\left[\int_{a}^{y}(y-t)^{n-1} g(t) d t\right]^{l} d y .
$$

From Eqs. (2.8) and (2.1), we have

$$
\mathrm{g}=\mathrm{W}(\mathrm{g})
$$

Let the set $Q_{r}:=\left\{g \in C([a, b], \mathbb{R}):\|g\|_{\infty}=\sup _{x \in[a, b]}|g(x)| \leqslant r\right\}$. The radius $r$ solves the nonlinear equation $|\lambda| k_{2}(b-a)\left(k_{3} r+k_{4}\right)^{p}-\left(|\mu|-k_{1}(b-a)\right) r+\|f\|_{\infty}+\|L\|_{\infty}=0$, where $k_{1}$ and $k_{2}$ are upper bounds for $|\Phi(x, t)|$ and $|\psi(x, y)|, \forall(x, t) \in[a, b]^{2}$ and $\forall(x, y) \in[a, b]^{2}$, respectively, $k_{3}:=\frac{(b-a)^{n}}{n !}$ and $k_{4}:=$ $\left|\sum_{j=0}^{n-1} \frac{(b-a)^{j}}{j !} q_{j}\right|$. First, we need to prove the operator $W$ maps the set $Q_{r}$ into itself as follows. Let $g \in Q_{r}$ be any function in this set. Then

$$
\begin{aligned}
|W(g)(x)| \leqslant & \frac{1}{|\mu|}|f(x)|+\frac{1}{|\mu|}|L(x)|+\frac{1}{|\mu|} \int_{a}^{x}|\Phi(x, t)||g(t)| d t \\
& +\frac{|\lambda|}{|\mu|} \int_{a}^{b} \sum_{l=0}^{p}|M(x, y ; l)|\left[\int_{a}^{y}(y-t)^{n-1}|g(t)| d t\right]^{l} d y \\
\leqslant & \frac{1}{|\mu|}\left[\|f\|_{\infty}+\|L\|_{\infty}+k_{1}(b-a) r\right] \\
& +\frac{|\lambda|}{|\mu|} \int_{a}^{b} \sum_{l=0}^{p} \frac{p !}{l !(p-l) !} \frac{r^{l}(b-a)^{n l}|\psi(x, y)|}{(n !)^{l}}\left|\sum_{j=0}^{n-1} \frac{(y-a)^{j}}{j !} q_{j}\right|^{p-l} d y .
\end{aligned}
$$

Since the function $\psi(x, y)$ is continuous on $[a, b]^{2}$. So, it is bounded and hence $|\psi(x, y)| \leqslant k_{2}, \forall(x, y) \in$ $[a, b]^{2}, k_{2}>0$,

$$
\begin{aligned}
|W(g)(x)| & \leqslant \frac{1}{|\mu|}\left[\|f\|_{\infty}+\|L\|_{\infty}+k_{1}(b-a) r\right] \\
& +\frac{|\lambda| k_{2}(b-a)}{|\mu|} \sum_{l=0}^{p} \frac{p !}{l !(p-l) !}\left(\frac{r(b-a)^{n}}{(n !)}\right)^{l}\left|\sum_{j=0}^{n-1} \frac{(b-a)^{j}}{j !} q_{j}\right|^{p-l} \\
& \leqslant \frac{1}{|\mu|}\left[\|f\|_{\infty}+\|L\|_{\infty}+k_{1}(b-a) r\right]+\frac{|\lambda| k_{2}(b-a)}{|\mu|}\left(\frac{r(b-a)^{n}}{(n !)}+\left|\sum_{j=0}^{n-1} \frac{(b-a)^{j}}{j !} q_{j}\right|\right)^{p} .
\end{aligned}
$$

Simplifying the above result implies

$$
\begin{aligned}
|W(g)(x)| & \leqslant \frac{1}{|\mu|}\left(\|f\|_{\infty}+\|L\|_{\infty}+k_{1}(b-a) r+|\lambda| k_{2}(b-a)\left(k_{3} r+k_{4}\right)^{p}\right) \\
& =\frac{1}{|\mu|}\left(\|f\|_{\infty}+\|L\|_{\infty}+k_{1}(b-a) r+|\mu| r-k_{1}(b-a) r-\|f\|_{\infty}-\|L\|_{\infty}\right)=r .
\end{aligned}
$$

Therefore, $\|W(g)\|_{\infty} \leqslant r$. Assume $\left\{x_{1}, x_{2}\right\} \in[a, b]$, and without loss of generality, let $x_{1}<x_{2}$. Suppose $g$ be an arbitrary function in the set $Q_{r}$.

$$
\begin{aligned}
& \left|W(g)\left(x_{2}\right)-W(g)\left(x_{1}\right)\right| \\
& \quad \leqslant \frac{1}{|\mu|}\left|f\left(x_{2}\right)-f\left(x_{1}\right)\right|+\frac{1}{|\mu|}\left|L\left(x_{2}\right)-L\left(x_{1}\right)\right|
\end{aligned}
$$




$$
\begin{aligned}
& +\frac{1}{|\mu|} \int_{a}^{x_{1}}\left|\Phi\left(x_{2}, t\right)-\Phi\left(x_{1}, t\right)\right||g(t)| d t+\frac{1}{|\mu|} \int_{x_{1}}^{x_{2}}\left|\Phi\left(x_{2}, t\right)\right||g(t)| d t \\
& +\frac{|\lambda|}{|\mu|} \int_{a}^{b} \sum_{l=0}^{p}\left|M\left(x_{2}, y ; l\right)-M\left(x_{1}, y ; l\right)\right|\left[\int_{a}^{y}(y-t)^{n-1}|g(t)| d t\right]^{l} d y \\
& \leqslant \frac{1}{|\mu|}\left|f\left(x_{2}\right)-f\left(x_{1}\right)\right|+\frac{1}{|\mu|}\left|L\left(x_{2}\right)-L\left(x_{1}\right)\right|+\frac{r}{|\mu|} \int_{a}^{x_{1}}\left|\Phi\left(x_{2}, t\right)-\Phi\left(x_{1}, t\right)\right| d t \\
& +\frac{r k_{1}}{|\mu|}\left(x_{2}-x_{1}\right)+\frac{|\lambda|}{|\mu|} \int_{a}^{b} \sum_{l=0}^{p}\left|M\left(x_{2}, y ; l\right)-M\left(x_{1}, y ; l\right)\right|\left[\int_{a}^{y}(y-t)^{n-1}|g(t)| d t\right]^{l} d y .
\end{aligned}
$$

But the functions $f, L, \Phi$ and $M$ are continuous. So, we have

$$
\begin{aligned}
\left|\mathrm{f}\left(\mathrm{x}_{2}\right)-\mathrm{f}\left(\mathrm{x}_{1}\right)\right| & \longrightarrow 0 \\
\left|\mathrm{~L}\left(\mathrm{x}_{2}\right)-\mathrm{L}\left(\mathrm{x}_{1}\right)\right| & \longrightarrow 0 \\
\left|\Phi\left(x_{2}, \mathrm{t}\right)-\Phi\left(x_{1}, \mathrm{t}\right)\right| & \longrightarrow 0 \\
\left|\mathrm{M}\left(\mathrm{x}_{2}, \mathrm{y} ; \mathrm{l}\right)-\mathrm{M}\left(\mathrm{x}_{1}, \mathrm{y} ; \mathrm{l}\right)\right| & \longrightarrow 0
\end{aligned}
$$

when $x_{2} \longrightarrow x_{1}$, and consequently $\left|W(g)\left(x_{2}\right)-W(g)\left(x_{1}\right)\right| \longrightarrow 0$. Therefore, the operator $W$ maps the set $Q_{r}$ into itself. Second, we need to prove that $W$ is a completely continuous operator (i.e., to show that the operator $W$ is continuous and $\left\{W\left(Q_{r}\right)\right\}$ is a compact set) as follows. Suppose a sequence $\left\{g_{n}\right\}_{n=1}^{\infty}$ with $g_{n} \in Q_{r}$, and assume $g_{n} \rightarrow g \in Q_{r}$, when $n \rightarrow \infty$. It is clear that $\left\|g_{n}\right\|_{\infty} \leqslant r, \forall n \in \mathbb{N}$.

$$
\begin{aligned}
& \left|W\left(g_{n}\right)(x)-W(g)(x)\right| \\
& \leqslant \frac{1}{|\mu|} \int_{a}^{x}|\Phi(x, t)|\left|g_{n}(t)-g(t)\right| d t \\
& \quad+\frac{|\lambda|}{|\mu|} \int_{a}^{b} \sum_{l=1}^{p}|M(x, y ; l)|\left|\left[\int_{a}^{y}(y-t)^{n-1} g_{n}(t) d t\right]^{l}-\left[\int_{a}^{y}(y-t)^{n-1} g(t) d t\right]^{l}\right| d y \\
& \leqslant \frac{1}{|\mu|} \int_{a}^{x}|\Phi(x, t)|\left|g_{n}(t)-g(t)\right| d t \\
& \quad+\frac{|\lambda|}{|\mu|} \int_{a}^{b} \sum_{l=1}^{p} c(l)|M(x, y ; l)|\left|\int_{a}^{y}(y-t)^{n-1} g_{n}(t) d t-\int_{a}^{y}(y-t)^{n-1} g(t) d t\right| d y, 0<c(l)<\infty \\
& \leqslant \\
& \frac{k_{1}}{|\mu|} \int_{a}^{x}\left|g_{n}(t)-g(t)\right| d t+\frac{|\lambda|}{|\mu|} \int_{a}^{b} \sum_{l=1}^{p} c(l)|M(x, y ; l)|\left(\int_{a}^{y}(y-t)^{n-1}\left|g_{n}(t)-g(t)\right| d t\right) d y .
\end{aligned}
$$

Applying the Arzela bounded convergence theorem implies

$$
\lim _{n \rightarrow \infty} \int_{a}^{x}\left|g_{n}(t)-g(t)\right| d t=\int_{a}^{x} \lim _{n \rightarrow \infty}\left|g_{n}(t)-g(t)\right| d t=0
$$

and

$$
\begin{aligned}
& \lim _{n \rightarrow \infty} \int_{a}^{b} \sum_{l=1}^{p} c(l)|M(x, y ; l)|\left(\int_{a}^{y}(y-t)^{n-1}\left|g_{n}(t)-g(t)\right| d t\right) d y \\
& \quad=\int_{a}^{b} \sum_{l=1}^{p} c(l)|M(x, y ; l)|\left(\int_{a}^{y}(y-t)^{n-1} \lim _{n \rightarrow \infty}\left|g_{n}(t)-g(t)\right| d t\right) d y=0 .
\end{aligned}
$$

So, $\left|W\left(g_{n}\right)(x)-W(g)(x)\right| \longrightarrow 0$, when $n \longrightarrow \infty$ and consequently the operator $W$ is continuous. Let $\left(W\left(g_{n}\right)\right)$ be a sequence with $g_{n} \in Q_{r}$. The sequence $\left(W\left(g_{n}\right)\right)$ is uniformaly bounded on $[a, b]$ because 
$\left\|\mathrm{W}\left(g_{\mathfrak{n}}\right)\right\|_{\infty} \leqslant r, \forall \mathfrak{n} \in \mathbb{N}$ from Ineq. (2.9). Furthermore, the sequence $\left(\mathrm{W}\left(\mathrm{g}_{\mathfrak{n}}\right)\right)$ is equicontinuous because $\left|W\left(g_{n}\right)\left(x_{2}\right)-W\left(g_{n}\right)\left(x_{1}\right)\right|<\epsilon$ as $\left|x_{2}-x_{1}\right|<\delta, \forall n \in \mathbb{N}$ from Ineq. (2.10). Applying the Arzela-Ascoli theorem implies that the sequence $\left(W\left(g_{n}\right)\right)$ contains a sub-sequence $\left(W\left(g_{n_{k}}\right)\right)$ which converges uniformly. So, the set $\left(W\left(Q_{r}\right)\right)$ is compact and hence $W$ is a completely continuous operator. From the Schauder fixed point theorem [4], Eq. (2.1) has at least one fixed point in $Q_{r}$ and therefore, the integro-differential Eq. (1.1) has at least one continuous solution. This completes the proof.

In what follows, we need to assume the next condition.

(v) Let $\frac{(b-a)\left[k_{1}+|\lambda| k_{2} k_{3} k_{5}\right]}{|\mu|}<1$, where $k_{5}:=\sum_{l=1}^{p}\left(\begin{array}{l}p \\ l\end{array}\right) \frac{c(l) k_{4}^{p-l}}{[(n-1) !]^{l-1}}$.

Theorem 2.4. Assume the conditions (i), (ii), (iv), and (v) are satisfied. Then the NF-VIE (2.1) has a unique continuous solution.

Proof. It is clear that Thm. 2.3 is satisfied from conditions (i), (ii), and (iv). So, Eq (2.1) has at least one continuous solution from applying Thm. 2.3. Now we need to prove that condition (v) must lead to the uniqueness of this solution. Let $g$ and $g^{*}$ be any two solutions of the NF-VIE (2.1). Then

$$
\begin{aligned}
\left|g(x)-g^{*}(x)\right| \leqslant & \frac{1}{|\mu|} \int_{a}^{x}|\Phi(x, t)|\left|g(x)-g^{*}(x)\right| d t \\
& +\frac{|\lambda|}{|\mu|} \int_{a}^{b} \sum_{l=1}^{p}|M(x, y ; l)|\left|\left[\int_{a}^{y}(y-t)^{n-1} g(t) d t\right]^{l}-\left[\int_{a}^{y}(y-t)^{n-1} g^{*}(t) d t\right]^{l}\right| d y \\
& \leqslant \frac{k_{1}(b-a)}{|\mu|}\left\|g-g^{*}\right\|_{\infty}+\frac{|\lambda| k_{2} k_{3}(b-a)}{|\mu|} \sum_{l=1}^{p} \frac{p ! c(l) k_{4}^{p-l}}{l !(p-l) ![(n-1) !]]^{l-1}}\left\|g-g^{*}\right\|_{\infty} \\
& \leqslant \frac{(b-a)\left[k_{1}+|\lambda| k_{2} k_{3} k_{5}\right]}{|\mu|}\left\|g-g^{*}\right\|_{\infty} .
\end{aligned}
$$

Passing supremun over $x \in[a, b]$ in both sides of Ineq. (2.11) yields

$$
\left(1-\frac{(b-a)\left[k_{1}+|\lambda| k_{2} k_{3} k_{5}\right]}{|\mu|}\right)\left\|g-g^{*}\right\|_{\infty} \leqslant 0
$$

but we have $\frac{(b-a)\left[k_{1}+|\lambda| k_{2} k_{3} k_{5}\right]}{|\mu|}<1$ from condition (v). So, we must have $\left\|g-g^{*}\right\|_{\infty}=0$ and hence $g=g^{*}$. This completes the proof.

\section{The modified Adomian's decomposition method}

In this section, we use the modified Adomian's decomposition method (MADM) to solve the NF-VIE (2.1). First, in the Adomain's decomposition method (ADM), the unknown function, $g(x)$, is decomposed into an infinite series of terms which can be determined in a recursive way through iterating, while the nonlinear part in Eq. (2.1) is decomposed into an infinite series of polynomials called the Adomian's polynomials, where these polynomials are not unique. Suppose the solution of Eq. (2.1) is given by the following infinite series

$$
g(x):=\sum_{m=0}^{\infty} g_{m}(x)
$$

Then, substituting in Eq. (2.1) yields

$$
\sum_{m=0}^{\infty} g_{m}(x)=\frac{1}{\mu} F(x)-\frac{1}{\mu} \int_{a}^{x} \Phi(x, t) \sum_{m=0}^{\infty} g_{m}(t) d t-\frac{\lambda}{\mu} \int_{a}^{b} \sum_{l=0}^{p} M(x, y ; l) \sum_{m=0}^{\infty} A_{m}(y ; l) d y,
$$


where $A_{m}, m \geqslant 0$ are the Adomain polynomials and given by

$$
A_{m}\left(g_{0}, g_{1}, \ldots, g_{m}, y ; l\right)=\left.\frac{1}{m !}\left(\frac{d^{m}}{d \rho^{m}}\left[\int_{a}^{y}(y-t)^{n-1} \sum_{i=0}^{\infty} \rho^{i} g_{i}(t) d t\right]^{l}\right)\right|_{\rho=0}, m=0,1,2, \ldots
$$

Set $g_{0}(x):=F(x)$, then we get the following recursive equation

$$
g_{0}(x)=\frac{1}{\mu} F(x), \quad g_{m}(x)=\frac{-1}{\mu} \int_{a}^{x} \Phi(x, t) g_{m-1}(t) d t-\frac{\lambda}{\mu} \int_{a}^{b} \sum_{l=0}^{p} M(x, y ; l) A_{m-1}(y ; l) d y, \quad m \geqslant 1,
$$

which can be used to determine the term $g_{m}, m \geqslant 1$. If the considered problem has a unique solution, then the series (3.1) will converge to that solution, see Thm. 3.1. Moreover, when the function $F(x)$ is the sum of two or more terms, it is better to use the MADM introduced by Wazwaz in [30] to accelerate the convergence rate and facilitate the calculations used to determine the generated terms as well. The recursive equation in this MADM is given as below

$$
\begin{aligned}
& g_{0}(x)=\frac{1}{\mu} F_{1}(x), \\
& g_{1}(x)=\frac{1}{\mu} F_{2}(x)-\frac{1}{\mu} \int_{a}^{x} \Phi(x, t) g_{0}(t) d t-\frac{\lambda}{\mu} \int_{a}^{b} \sum_{l=0}^{p} M(x, y ; l) A_{0}(y ; l) d y, \\
& g_{m}(x)=-\frac{1}{\mu} \int_{a}^{x} \Phi(x, t) g_{m-1}(t) d t-\frac{\lambda}{\mu} \int_{a}^{b} \sum_{l=0}^{p} M(x, y ; l) A_{m-1}(y ; l) d y, \quad m \geqslant 2,
\end{aligned}
$$

where $F(x):=F_{1}(x)+F_{2}(x)$. As we can see, the difference between the ADM and the MADM rests in the construction of the first two terms $g_{0}$ and $g_{1}$ only.

Theorem 3.1. Let the conditions of Thm. 2.4 are verified. Then the infinite series solution $\sum_{m=0}^{\infty} g_{m}(x)$ of the NF-VIE (2.1) converges to the exact solution $\mathrm{g}(\mathrm{x})$.

Proof. Let $m$ and $n$ be two distinct positive integers with $m>n \geqslant 1$. Suppose we have the following sequence of partial sums $\left(S_{m}(x)\right)$, where $S_{m}(x)=\sum_{i=0}^{m} g_{i}(x)$. Then

$$
\begin{aligned}
\left|S_{m}(x)-S_{n}(x)\right|=\left|\sum_{i=n+1}^{m} g_{i}(x)\right| & \leqslant \frac{1}{|\mu|} \int_{a}^{x}|\Phi(x, t)|\left|\sum_{i=n}^{m-1} g_{i}(t)\right| d t+\frac{|\lambda|}{|\mu|} \int_{a}^{b} \sum_{l=0}^{p}|M(x, y ; l)|\left|\sum_{i=n}^{m-1} A_{i}(y ; l)\right| d y \\
& \leqslant \frac{k_{1}(b-a)}{|\mu|}\left\|S_{m-1}-S_{n-1}\right\|_{\infty}+\frac{|\lambda|}{|\mu|} \int_{a}^{b} \sum_{l=0}^{p}|M(x, y ; l)|\left|\sum_{i=n}^{m-1} A_{i}(y ; l)\right| d y .
\end{aligned}
$$

Since

$$
\begin{aligned}
\left|\sum_{i=n}^{m-1} A_{i}(y ; l)\right| & =\left|\sum_{i=0}^{m-1} A_{i}(y ; l)-\sum_{i=0}^{n-1} A_{i}(y ; l)\right| \\
& =\left|\left[\int_{a}^{y}(y-t)^{n-1} \sum_{i=0}^{m-1} g_{i}(t) d t\right]^{l}-\left[\int_{a}^{y}(y-t)^{n-1} \sum_{i=0}^{n-1} g_{i}(t) d t\right]^{l}\right| \\
& \leqslant c(l)\left|\int_{a}^{y}(y-t)^{n-1} \sum_{i=0}^{m-1} g_{i}(t) d t-\int_{a}^{y}(y-t)^{n-1} \sum_{i=0}^{n-1} g_{i}(t) d t\right|, 0<c(l)<\infty \\
& \leqslant c(l)\left\|S_{m-1}-S_{n-1}\right\|_{\infty} \int_{a}^{y}(y-t)^{n-1} d t \\
& \leqslant \frac{c(l)(b-a)^{n}}{n}\left\|S_{m-1}-S_{n-1}\right\|_{\infty} .
\end{aligned}
$$


Then, substituting Eq. (3.3) in Eq. (3.2) yields

$$
\left|S_{m}(x)-S_{n}(x)\right| \leqslant \frac{(b-a)\left[k_{1}+|\lambda| k_{2} k_{3} k_{5}\right]}{|\mu|}\left\|S_{m-1}-S_{n-1}\right\|_{\infty}
$$

Let $\gamma:=\frac{(b-a)\left[k_{1}+|\lambda| k_{2} k_{3} k_{5}\right]}{|\mu|}$, where $\gamma<1$ from Thm. 2.4. Then we have

$$
\left\|S_{m}-S_{n}\right\|_{\infty} \leqslant \gamma\left\|S_{m-1}-S_{n-1}\right\|_{\infty}
$$

Let $m=n+1$. So, we have

$$
\left\|S_{n+1}-S_{n}\right\|_{\infty} \leqslant \gamma^{n}\left\|g_{1}\right\|_{\infty}
$$

Substituting Ineq. (3.5) in Ineq. (3.4) after applying the triangle inequality and letting $m \gg n>N \in \mathbb{N}$, we obtain

$$
\left\|S_{m}-S_{n}\right\|_{\infty} \leqslant \frac{\gamma^{n}}{1-\gamma}\left\|g_{1}\right\|_{\infty}=\epsilon,
$$

where $\lim _{n \rightarrow \infty} \gamma^{n}=0$. So, the sequence $\left(S_{n}(x)\right)$ is a Cauchy sequence in the Banach space $C([a, b], \mathbb{R})$. Therefore $\lim _{n \rightarrow \infty} S_{n}(x)=g(x)$ because the solution of Eq. (2.1) is unique under conditions of Thm. 2.4. This completes the proof.

Lemma 3.2. The truncation error between the exact solution, $g(x)$, of the NF-VIE (2.1) and approximate solution, $S_{n}(x)=\sum_{i=0}^{n} g_{i}(x)$, is estimated by $\left\|g-S_{n}\right\|_{\infty} \leqslant \frac{\gamma^{n}}{1-\gamma}\left\|g_{1}\right\|_{\infty}$.

Proof. The proof follows directly from Thm. 3.1. So, it is omitted.

\section{The homotopy analysis method}

In this section, the homotopy analysis method (HAM) is applied to solve the NF-VIE (2.1) under satisfying conditions of Thm. 2.4. From Eq. (2.1), we have

$$
\mu g(x)-F(x)+\int_{a}^{x} \Phi(x, t) g(t) d t+\lambda \int_{a}^{b} \sum_{l=0}^{p} M(x, y ; l)\left[\int_{a}^{y}(y-t)^{n-1} g(t) d t\right]^{l} d y=0 .
$$

Define the operator $\mathrm{N}$ by

$$
N[g(x)]=g(x)-\frac{1}{\mu} F(x)+\frac{1}{\mu} \int_{a}^{x} \Phi(x, t) g(t) d t+\frac{\lambda}{\mu} \int_{a}^{b} \sum_{l=0}^{p} M(x, y ; l)\left[\int_{a}^{y}(y-t)^{n-1} g(t) d t\right]^{l} d y .
$$

From (4.1) and (4.2) we have

$$
\mathrm{N}[\mathrm{g}(\mathrm{x})]=0, \quad x \in[\mathrm{a}, \mathrm{b}] .
$$

We define the homotopy of the unknown function $g(x)$ as below

$$
\mathcal{H}^{*}[\Psi(x ; \hbar, p)]:=(1-p) \mathcal{L}\left[\Psi(x ; \hbar, p)-g_{0}(x)\right]-p \hbar N[\Psi(x ; \hbar, p)],
$$

(1) the function $g_{0}(x)$ is the initial approximation of the unknown solution $g(x)$;

(2) the parameter $\hbar \in \mathbb{R} \backslash\{0\}$ is used as a control tool to manage the convergence of the proposed technique;

(3) the parameter $p \in[0,1]$ is embedding in Eq. (4.4) and called the homotopy parameter; 
(4) the operator $\mathcal{L}$ is an auxiliary linear operator satisfying the property $\mathcal{L}[\mathbf{r}(x)]=0$ when $\mathbf{r}(\mathrm{x})=0$;

(5) the operator $\mathrm{N}$ denotes Eq. (4.2), that is

$$
\begin{aligned}
N[\Psi(x ; \hbar, p)]= & \Psi(x ; \hbar, p)-\frac{1}{\mu} F(x)+\frac{1}{\mu} \int_{a}^{x} \Phi(x, t) \Psi(t ; \hbar, p) d t \\
& +\frac{\lambda}{\mu} \int_{a}^{b} \sum_{l=0}^{p} M(x, y ; l)\left[\int_{a}^{y}(y-t)^{n-1} \Psi(t ; \hbar, p) d t\right]^{l} d y .
\end{aligned}
$$

Solving Eq. (4.5)

$$
\mathcal{H}^{*}[\Psi(x ; \hbar, p)]=0,
$$

yields

$$
(1-p) \mathcal{L}\left[\Psi(x ; \hbar, p)-g_{0}(x)\right]=p \hbar N[\Psi(x ; \hbar, p)],
$$

where Eq. (4.6) is called the zero-order deformation. Define the operator $\mathcal{L}$ in the current work by $\mathcal{L}[\mathrm{g}]:=\mathrm{g}$. So, the zero-order deformation in this work is defined as

$$
(1-p)\left[\Psi(x ; \hbar, p)-g_{0}(x)\right]=p \hbar N[\Psi(x ; \hbar, p)] .
$$

Remark 4.1. From Eq. (4.5), we can see the following two important notes.

(1) substituting $p=0$ in Eq. (4.5), and then using the property of the operator $\mathcal{L}$ imply

$$
\mathcal{H}^{*}[\Psi(x ; \hbar, 0)]=0 \Longrightarrow \mathcal{L}\left[\Psi(x ; \hbar, 0)-g_{0}(x)\right]=0 \Longrightarrow \Psi(x ; \hbar, 0)=g_{0}(x),
$$

where $g_{0}(x)$ is the initial guess of the unknown solution $g(x)$, so, $\Psi(x ; \hbar, 0)=g_{0}(x)$ is a solution for Eq. (4.5) which is obtained when $p=0$;

(2) substituting $p=1$ in Eq. (4.5) gives

$$
\mathcal{H}^{*}[\Psi(x ; \hbar, 1)]=0 \Longrightarrow-\hbar N[\Psi(x ; \hbar, 1)]=0 \Longrightarrow N[\Psi(x ; \hbar, 1)]=0,
$$

but from Eq. (4.3) we have $N[g(x)]=0$ and consequently $\Psi(x ; \hbar, 1)=g(x)$, so, $\Psi(x ; \hbar, 1)=g(x)$ is a solution for Eq. (4.5) which is obtained when $p=1$.

From remark (4.1), the essence of the HAM technique turns out to be: "while the homotopy-parameter, $p$, is increasing from 0 to 1 , the homoptopy function, $\Psi(x ; \hbar, p)$, varies continuously from the initial approximation, $g_{0}(x)$, to the solution $g(x)$ of Eq. (2.1), see [21]".

Let the control parameter, $\hbar$, be selected so that the solution of Eq. $(4.7)$ exists for $p \in(0,1)$ and is analytic at $p=0$ [21]. So, we can assume the solution of Eq. (2.1) on the form

$$
g(x)=g_{0}(x)+\sum_{m=1}^{\infty} g_{m}(x)=\sum_{m=0}^{\infty} g_{m}(x),
$$

where

$$
g_{m}(x)=\left.\frac{1}{m !} \frac{\partial^{m} \Psi(x ; \hbar, p)}{\partial p^{m}}\right|_{p=0}, \quad m=1,2,3,4, \ldots
$$

The recursive equation for generating the individual terms of the proposed series solution (4.8), which is obtained by differentiating both sides of Eq. (4.7) m-times with respect to the parameter $p$ and then substituting $p=0$, is given by

$$
g_{1}(x)=\hbar \mathbf{R}_{1}\left(g_{0}(x)\right), \quad g_{m}(x)=g_{m-1}(x)+\hbar \mathbf{R}_{m}\left(\bar{g}_{m-1}(x)\right), \quad m \geqslant 2,
$$

where

$$
\begin{aligned}
\overline{\mathbf{g}}_{\mathrm{m}-1}(x) & =\left(g_{0}(x), g_{1}(x), \cdots, g_{m-1}(x)\right) \\
\mathbf{R}_{\mathrm{m}}\left(\overline{\mathbf{g}}_{\mathrm{m}-1}(x)\right) & =\left.\frac{1}{(m-1) !}\left[\frac{\partial^{m-1}}{\partial p^{m-1}} \mathrm{~N}\left(\sum_{i=0}^{\infty} g_{i}(x) p^{i}\right)\right]\right|_{p=0}
\end{aligned}
$$




\section{Analytical and numerical results}

Example 5.1. Consider the second order nonlinear integro-differential equation

$$
2 \phi^{\prime \prime}(x)+x^{2} \phi^{\prime}(x)-2 x \phi(x)=\frac{-7}{12} x-\frac{1}{20} x^{4}-\frac{160}{3} \int_{0}^{1} x y[\phi(y)]^{2} d y, 0 \leqslant x \leqslant 1,
$$

subject to the initial conditions

$$
\phi(0)=0, \phi^{\prime}(0)=0
$$

Using Eq. (2.4) we get on

$$
\phi(x)=\int_{0}^{x}(x-t) g(t) d t
$$

where $g(x)$ is the solution of the following NF-VIE

$$
2 g(x)+\int_{0}^{x}\left(2 x t-x^{2}\right) g(t) d t=\frac{-7}{12} x-\frac{1}{20} x^{4}-\frac{160}{3} \int_{0}^{1} x y\left[\int_{0}^{y}(y-t) g(t) d t\right]^{2} d y .
$$

The exact solution for Eq. (5.3) is $g(x)=\frac{-3}{10} x$ and consequently substituting in Eq. (5.2) yields $\phi(x)=\frac{-1}{20} x^{3}$ which is the exact solution for Eq. (5.1).

It is worth mentioning that this solution $g(x)=\frac{-3}{10} x$ of Eq. (5.3) is unique. Indeed, it is clear that the functions $B_{1}(x)=x^{2}$ and $B_{2}(x)=-2 x$ satisfy condition (i). The functions $f(x)=\frac{-7}{12} x-\frac{1}{20} x^{4}$ and $\psi(x, y)=x y$ verify conditions (ii) and (iv), respectively. It is easy to figure out that $\mu=2, \lambda=\frac{160}{3}, k_{1}=$ $\|\Phi(x, t)\|_{\infty}=\left\|2 x t-x^{2}\right\|_{\infty}=1$ where $0 \leqslant t \leqslant x \leqslant 1$. Also, we have $k_{2}=\|\psi(x, y)\|_{\infty}=\|x y\|_{\infty}=1$ where $(x, y) \in[0,1]^{2}, k_{3}=\frac{1}{2}, k_{4}=k_{5}=0$. Finally, condition (v) is satisfied as well because $\frac{(b-a)\left[k_{1}+|\lambda| k_{2} k_{3} k_{5}\right]}{|\mu|}=$ $\frac{1}{2}<1$. Hence Eq. (5.3) has a unique solution from applying Thm. 2.4.

5.1. Applying the MADM.

Set

$$
\begin{aligned}
g_{0}(x) & =\frac{-7}{24} x, \\
g_{1}(x) & =\frac{-1}{40} x^{4}-\frac{1}{2} \int_{0}^{x}\left(2 x t-x^{2}\right) g_{0}(t) d t-\frac{80}{3} \int_{0}^{1} x y A_{0}(y) d y, \\
g_{m}(x) & =\frac{-1}{2} \int_{0}^{x}\left(2 x t-x^{2}\right) g_{m-1}(t) d t-\frac{80}{3} \int_{0}^{1} x y A_{m-1}(y) d y, m \geqslant 2,
\end{aligned}
$$

where the Adomain polynomials, $A_{m}, m \geqslant 0$, are given by

$$
A_{m}\left(g_{0}, g_{1}, \ldots, g_{m}, y\right)=\left.\frac{1}{m !}\left(\frac{d^{m}}{d \rho^{m}}\left[\int_{0}^{y}(y-t) \sum_{i=0}^{\infty} \rho^{i} g_{i}(t) d t\right]^{2}\right)\right|_{\rho=0}, m=0,1,2, \ldots
$$

Then, applying the recursive Eq. (5.4) after generating the Adomian polynomials from Eq. (5.5) implies

$$
\begin{aligned}
& g_{0}(x)=\frac{-7}{24} x, \\
& g_{1}(x)=\frac{-1}{1440} x^{4}-\frac{245}{31104} x, \\
& g_{2}(x)=\frac{1}{21600} x^{7}+\frac{245}{373248} x^{4}-\frac{477673}{1108546560} x,
\end{aligned}
$$


We demonstrate in Tables 1 and 2 a comparison between exact solution and approximate solutions $S_{1}(x), S_{2}(x), S_{3}(x), S_{4}(x), S_{5}(x)$, and $S_{6}(x)$, respectively using the MADM in Ex. 5.1. Also we present in these tables the corresponding infinite norm of the absolute errors in each approximation. We can realize from the numerical data in these two Tables 1 and 2 that the infinite norm of the errors decreases as the partial sum of the approximated terms increases. Also, the approximate solution using the MADM converges very rapidly to the exact solution which is clear from Fig. 1.

\subsection{Applying the HAM.}

From Eq. (5.3), we define the operator $\mathrm{N}$ by

$$
\begin{aligned}
N[\Psi(x ; \hbar, p)]= & \Psi(x ; \hbar, p)+\frac{7}{24} x+\frac{1}{40} x^{4}+\frac{1}{2} \int_{0}^{x}\left(2 x t-x^{2}\right) \Psi(t ; \hbar, p) d t \\
& +\frac{80}{3} \int_{0}^{1} x y\left[\int_{0}^{y}(y-t) \Psi(t ; \hbar, p) d t\right]^{2} d y .
\end{aligned}
$$

Using Eq. (4.7), we define the zero-order deformation as below

$$
(1-p)\left[\Psi(x ; \hbar, p)+\frac{7}{24} x\right]=p \hbar N[\Psi(x ; \hbar, p)]
$$

where $g_{0}(x)=\frac{-7}{24} x$. It is clear that when $p=0$, and $p=1$ we have $\Psi(x ; \hbar, 0)=\frac{-7}{24} x$ and $\Psi(x ; \hbar, 1)=g(x)$. Using the recursive Eq. (4.9) and Eq. (4.10) we get the individual terms of the series solution (4.8) as follows:

$$
\begin{aligned}
& g_{0}(x)=\frac{-7}{24} x, \\
& g_{1}(x)=\hbar\left(\frac{1}{1440} x^{4}+\frac{245}{31104} x\right) \\
& g_{2}(x)=\frac{1}{21600} \hbar^{2} x^{7}+\left(\frac{2521}{1866240} \hbar+\frac{1}{1440}\right) \hbar x^{4}+\left(\frac{8254127}{1108546560} \hbar+\frac{245}{31104}\right) \hbar x,
\end{aligned}
$$

The proper numbers of the control parameter $\hbar$ which guarantee the convergence of the approximate series solution to the exact solution are determined from the line segments which are almost parallel to the horizontal $\hbar$-axis in the $\hbar$-curves in Fig. 2 . The value of the control parameter $\hbar$ which minimizes the squared of the residual is called the optimal value, see Fig. 3. For example, $\hbar \simeq$ $-1.012033,-1.00463,-1.01064,-1.01115,-1.01561,-1.02019$ are the values which minimize the squared residual based on utilizing $S_{1}(x), S_{2}(x), S_{3}(x), S_{4}(x), S_{5}(x)$, and $S_{6}(x)$, respectively, where $x \in[0,1]$, see Fig. 3. When the value of $\hbar=-1$, we get the same numerical results obtained using the MADM as in Tables 1 and 2. Therefore, based on the numerical results we obtained in Tables 3 and 4 , it is very convenient to take $\hbar=-1$ to be the optimal value for the control parameter $\hbar$ in Ex. 5.1. Therefore, no significance difference was detected between the MADM and HAM during applying the two methods to solve Ex. 5.1 provided we use the same initial approximation in both MADM and HAM.

Example 5.2. Consider the third order nonlinear integro-differential equation:

$$
\begin{aligned}
& \frac{9}{2} \phi^{\prime \prime \prime}(x)-x^{2} \phi^{\prime \prime}(x)-x^{2} \phi^{\prime}(x)+\frac{9}{2} \phi(x) \\
& \quad=\frac{9}{2} x+\left(\frac{640}{231} e^{-1}-\frac{25}{231} e^{-2}-2\right) x^{2}-\frac{20}{231} \int_{0}^{1} x^{2} y^{2}[\phi(y)]^{2} d y, 0 \leqslant x \leqslant 1,
\end{aligned}
$$


subject to the initial conditions

$$
\phi(0)=-1, \phi^{\prime}(0)=2, \phi^{\prime \prime}(0)=-1 \text {. }
$$

Using Eq. (2.4) we get on

$$
\phi(x)=-1+2 x-\frac{x^{2}}{2 !}+\int_{0}^{x} \frac{(x-t)^{2}}{2 !} g(t) d t,
$$

where $g(x)$ is the solution of the following NF-VIE

$$
\begin{aligned}
& \frac{9}{2} g(x)+\int_{0}^{x}\left[\frac{9}{4} t^{2}+\left(x^{2}-\frac{9}{2} x\right) t-\left(x^{3}-\frac{5}{4} x^{2}\right)\right] g(t) d t \\
& =\frac{9}{2}(1-x)+\left(\frac{2722}{2183}+\frac{640 e^{-1}}{231}-\frac{25 e^{-2}}{231}\right) x^{2}-x^{3} \\
& \quad+\frac{20}{231} \int_{0}^{1} \int_{0}^{y} x^{2}\left(\frac{1}{2} y^{4}-2 y^{3}+y^{2}\right)(y-t)^{2} g(t) d t d y-\frac{5}{231} \int_{0}^{1} x^{2} y^{2}\left[\int_{0}^{y}(y-t)^{2} g(t) d t\right]^{2} d y .
\end{aligned}
$$

The exact solution for Eq. (5.8) is $g(x)=e^{-x}$ and consequently substituting in Eq. (5.7) yields $\phi(x)=$ $x-e^{-x}$ which is the exact solution for Eq. (5.6).

The solution $g(x)=e^{-x}$ of Eq. (5.8) is unique. Indeed, it is clear that the functions $B_{1}(x)=$ $-x^{2}, B_{2}(x)=-x^{2}$, and $B_{3}(x)=\frac{9}{2}$ satisfy condition (i). The functions $f(x)=\frac{9}{2} x+\left(\frac{640}{231} e^{-1}-\frac{25}{231} e^{-2}-2\right) x^{2}$ and $\psi(x, y)=x^{2} y^{2}$ verify conditions (ii) and (iv), respectively. It is easy to figure out that $\mu=\frac{9}{2}, \lambda=$ $\frac{20}{231}, k_{1}=\|\Phi(x, t)\|_{\infty}=\left\|\frac{9}{4} t^{2}+\left(x^{2}-\frac{9}{2} x\right) t-\left(x^{3}-\frac{5}{4} x^{2}\right)\right\|_{\infty}=\frac{10}{9}$, where $0 \leqslant t \leqslant x \leqslant 1$. Also, we have $k_{2}=\|\psi(x, y)\|_{\infty}=\left\|x^{2} y^{2}\right\|_{\infty}=1$, where $(x, y) \in[0,1]^{2}, k_{3}=\frac{1}{6}, k_{4}=\frac{1}{2}, k_{5}=\frac{741}{413}$. Also, condition $(v)$ is satisfied because $\frac{(b-a)\left[k_{1}+|\lambda| k_{2} k_{3} k_{5}\right]}{|\mu|}=\frac{1279}{2531}<1$. So, Eq. (5.3) has a unique solution from applying Thm. 2.4 .

\subsection{Applying the MADM.}

Set

$$
\begin{aligned}
g_{0}(x)= & 1-x \\
g_{1}(x)= & \frac{2}{9}\left(\frac{2722}{2183}+\frac{640 e^{-1}}{231}-\frac{25 e^{-2}}{231}\right) x^{2}-\frac{2}{9} x^{3}-\frac{2}{9} \int_{0}^{x}\left[\frac{9}{4} t^{2}+\left(x^{2}-\frac{9}{2} x\right) t-\left(x^{3}-\frac{5}{4} x^{2}\right)\right] g_{0}(t) d t \\
& +\frac{40}{2079} \int_{0}^{1} \int_{0}^{y} x^{2}\left(\frac{1}{2} y^{4}-2 y^{3}+y^{2}\right)(y-t)^{2} g_{0}(t) d t d y-\frac{10}{2079} \int_{0}^{1} x^{2} y^{2} A_{0}(y) d y, \\
g_{m}(x)= & -\frac{2}{9} \int_{0}^{x}\left[\frac{9}{4} t^{2}+\left(x^{2}-\frac{9}{2} x\right) t-\left(x^{3}-\frac{5}{4} x^{2}\right)\right] g_{m-1}(t) d t \\
& +\frac{40}{2079} \int_{0}^{1} \int_{0}^{y} x^{2}\left(\frac{1}{2} y^{4}-2 y^{3}+y^{2}\right)(y-t)^{2} g_{m-1}(t) d t d y \\
& -\frac{10}{2079} \int_{0}^{1} x^{2} y^{2} A_{m-1}(y) d y, m \geqslant 2,
\end{aligned}
$$

where the Adomain polynomials, $A_{m}, m \geqslant 0$, are given by

$$
A_{m}\left(g_{0}, g_{1}, \cdots, g_{m}, y\right)=\left.\frac{1}{m !}\left(\frac{d^{m}}{d \rho^{m}}\left[\int_{0}^{y}(y-t)^{2} \sum_{i=0}^{\infty} \rho^{i} g_{i}(t) d t\right]^{2}\right)\right|_{\rho=0}, m=0,1,2, \ldots
$$

Then, applying the recursive Eq. (5.9) after generating the Adomian polynomials from Eq. (5.10) implies 


$$
\begin{aligned}
g_{0}(x)= & 1-x, \\
g_{1}(x)= & -\frac{1}{27} x^{5}+\frac{1}{24} x^{4}-\frac{1}{6} x^{3}+\frac{58398847732950836625617}{116793857742032880009216} x^{2}, \\
g_{2}(x)= & -\frac{1}{5103} x^{9}-\frac{389}{408240} x^{8}-\frac{1}{5040} x^{7}+\frac{43807290962934313108501}{31534341590348877602488320} x^{6} \\
& +\frac{1810364279721475935394127}{63068683180697755204976640} x^{5}-\frac{202262438675599846728213691}{12500086869047933686115960094720} x^{2},
\end{aligned}
$$

Tables 5 and 6 demonstrate a comparison between exact solution $g(x)=e^{-x}$ in Ex. 5.2 and approximate solutions $S_{1}(x), S_{2}(x), S_{3}(x), S_{4}(x), S_{5}(x)$, and $S_{6}(x)$, respectively. From Fig. 4 and the numerical data in Tables 5 and 6 we notice that the infinite norm of errors decreases when the partial sum of terms increases. Also, the approximate solution converges very fast to the exact solution.

\subsection{Applying the HAM}

From Eq. (5.8), we define the operator $\mathrm{N}$ by

$$
\begin{aligned}
\mathrm{N}[\Psi(x ; \hbar, p)]= & \Psi(x ; \hbar, p)-1+x-\frac{2}{9}\left(\frac{2722}{2183}+\frac{640 e^{-1}}{231}-\frac{25 e^{-2}}{231}\right) x^{2}+\frac{2}{9} x^{3} \\
& +\frac{2}{9} \int_{0}^{x}\left[\frac{9}{4} t^{2}+\left(x^{2}-\frac{9}{2} x\right) t-\left(x^{3}-\frac{5}{4} x^{2}\right)\right] \Psi(t ; \hbar, p) d t \\
& -\frac{40}{2079} \int_{0}^{1} \int_{0}^{y} x^{2}\left(\frac{1}{2} y^{4}-2 y^{3}+y^{2}\right)(y-t)^{2} \Psi(t ; \hbar, p) d t d y \\
& +\frac{10}{2079} \int_{0}^{1} x^{2} y^{2}\left[\int_{0}^{y}(y-t)^{2} \Psi(t ; \hbar, p) d t\right]^{2} d y .
\end{aligned}
$$

Using Eq. (4.7), we define the zero-order deformation as below

$$
(1-p)[\Psi(x ; \hbar, p)-1+x]=p \hbar N[\Psi(x ; \hbar, p)],
$$

where $g_{0}(x)=1-x$. It is clear that when $p=0$, and $p=1$ we have $\Psi(x ; \hbar, 0)=1-x$ and $\Psi(x ; \hbar, 1)=g(x)$. Using the recursive Eq. (4.9) and Eq. (4.10) we get the individual terms of the series solution (4.8) as below

$$
\begin{aligned}
g_{0}(x)= & 1-x, \\
g_{1}(x)= & \hbar\left(\frac{1}{27} x^{5}-\frac{1}{24} x^{4}+\frac{1}{6} x^{3}-\frac{58398847732950836625617}{116793857742032880009216} x^{2}\right), \\
g_{2}(x)= & \hbar^{2}\left(\frac{-1}{5103} x^{9}-\frac{389}{408240} x^{8}-\frac{1}{5040} x^{7}+\frac{43807290962934313108501}{31534341590348877602488320} x^{6}\right) \\
& +\hbar\left(\frac{4146241434562133535578447}{63068683180697755204976640} \hbar+\frac{1}{27}\right) x^{5}-\frac{1}{24} \hbar(\hbar+1) x^{4}+\frac{1}{6} \hbar(\hbar+1) x^{3} \\
& -\hbar\left(\frac{6250451066845386155309708823331}{12500086869047933686115960094720} \hbar+\frac{58398847732950836625617}{116793857742032880009216}\right) x^{2},
\end{aligned}
$$

As we did in the previous example, we can depict from the $\hbar$-curves in Fig. 5 the values of the parameter $\hbar$ which guarantee the convergence of the series solution to the exact solution. Also, the optimal value of the control parameter $\hbar$ corresponding to each approximation are illustrated in Fig. 6. For example, $\hbar \simeq-1.049902,-1.019211,-1.011092,-1.015997,-1.014091,-1.006894$ are the optimal values corresponding to $S_{1}(x), S_{2}(x), S_{3}(x), S_{4}(x), S_{5}(x)$, and $S_{6}(x)$, respectively, where $x \in[0,1]$. We confirm that the two 
proposed methods shows the same numerical results when we substitute $\hbar=-1$ in the HAM. Comparing Tables 7 and 8 with Tables 5 and 6, we can see that applying the HAM gives an approximate solution which converges slightly faster and more accurate than the one obtained through using the MADM.

\section{Figures}

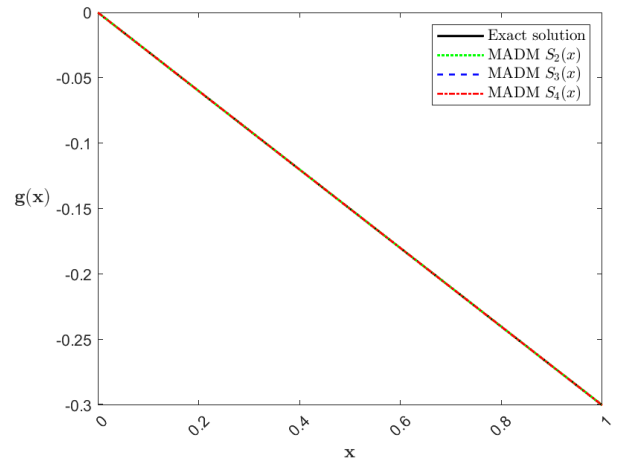

Figure 1: Depiction of the exact solution $g(x)=\frac{-3}{10} x$ along with three approximate solutions $S_{2}(x), S_{3}(x)$, and $S_{4}(x)$ using the MADM in Ex. 5.1.

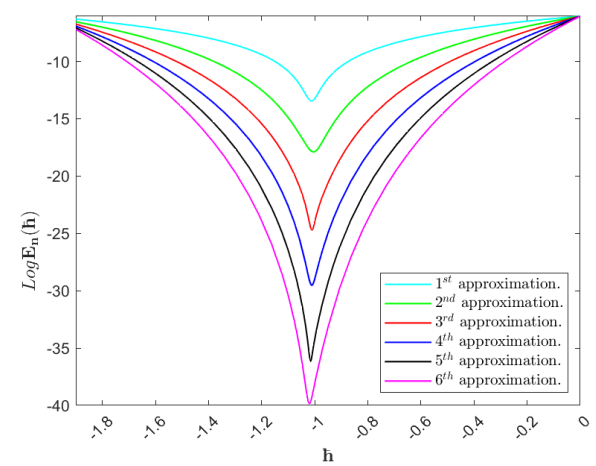

Figure 3: Depiction of the natural logarithm of squared residual, $\mathbf{E}_{\mathbf{n}}$ when $\mathrm{n}=1,2,3,4,5,6$ to visualize the corresponding optimal value of the convergence control parameter $\hbar$ in HAM in Ex. 5.1.

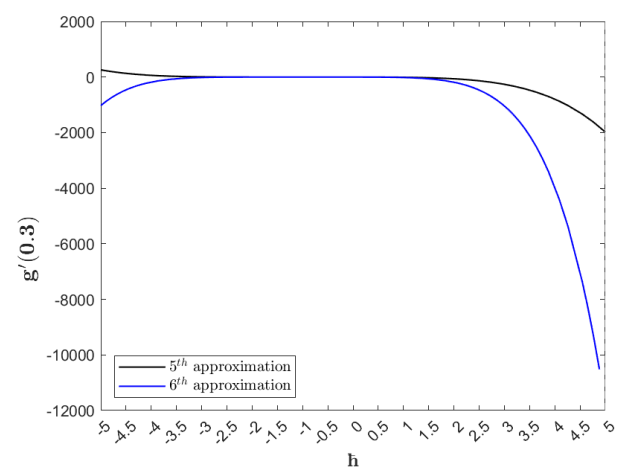

Figure 5: The $\hbar$-curves of $g /(0.3)$ in Ex. 5.2 derived from the $5^{\text {th }}$ and $6^{\text {th }}$ approximations using HAM.

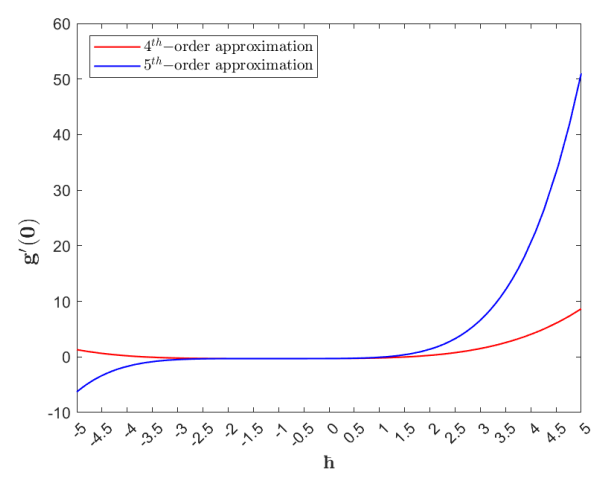

Figure 2: The $\hbar$-curves of $g /(0)$ in Ex. 5.1 derived from the $4^{\text {th }}$ and $5^{\text {th }}$ approximations using HAM.

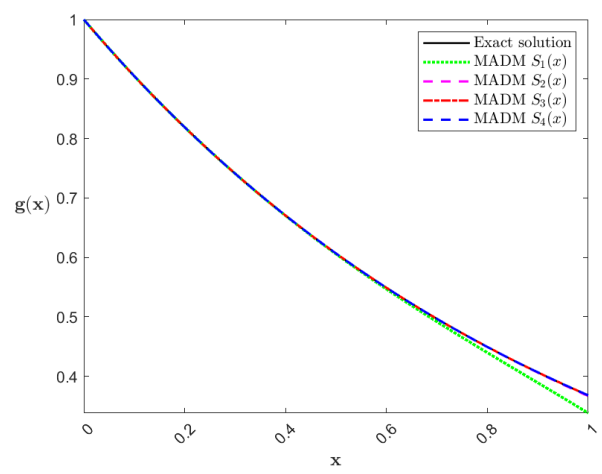

Figure 4: Depiction of the exact solution $g(x)=e^{-x}$ along with four approximate solutions $S_{1}(x), S_{2}(x), S_{3}(x)$, and $S_{4}(x)$ using the MADM in Ex. 5.2.

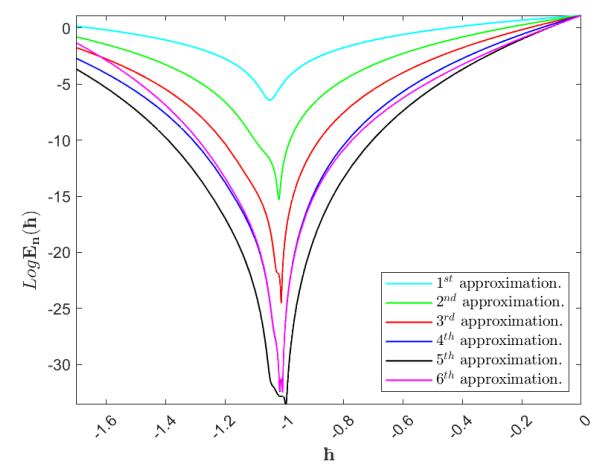

Figure 6: Depiction of the natural logarithm of squared residual when $n=1,2,3,4,5,6$ to visualize the corresponding optimal value of the convergence control parameter $\hbar$ in HAM in Ex. 5.2. 


\section{Tables}

Table 1: Exact solution $g(x)=\frac{-3}{10} x$ and approximate solutions $S_{1}(x), S_{2}(x)$, and $S_{3}(x)$ utilizing the MADM along with the corresponding infinite norm of the absolute errors in Ex. 5.1.

\begin{tabular}{|c|c|c|c|c|c|c|c|}
\hline $\mathrm{x}$ & Exact solution & ${\text { MADM } S_{1}(\mathrm{x})}$ & $\left|\mathrm{E}_{1}\right|$ & $\mathrm{MADM}_{2}(\mathrm{x})$ & $\left|\mathrm{E}_{2}\right|$ & ${\text { MADM } S_{3}(\mathrm{x})}_{|c|}\left|\mathrm{E}_{3}\right|$ \\
\hline 0.0 & 0.0 & 0.0 & 0.0 & 0.0 & 0.0 & 0.0 & 0.0 \\
\hline 0.2 & -0.06 & -0.0599098045 & 0.0000901954 & -0.0599949337 & 0.0000050662 & -0.0599996550 & 0.0000003449 \\
\hline 0.4 & -0.12 & -0.1198351646 & 0.0001648353 & -0.1199906450 & 0.0000093549 & -0.1199993541 & 0.0000006458 \\
\hline 0.6 & -0.18 & -0.1798160802 & 0.0001839197 & -0.1799882549 & 0.0000117450 & -0.1799991745 & 0.0000008254 \\
\hline 0.8 & -0.24 & -0.2399192181 & 0.0000807818 & -0.2399853678 & 0.0000146321 & -0.2399991914 & 0.0000008085 \\
\hline 1.0 & -0.30 & -0.3002379115 & 0.0002379115 & -0.2999661154 & 0.0000338845 & -0.3000001087 & 0.0000001087 \\
\hline
\end{tabular}

Table 2: Exact solution $g(x)=\frac{-3}{10} x$ and approximate solutions $S_{4}(x), S_{5}(x)$, and $S_{6}(x)$ utilizing the MADM along with the corresponding infinite norm of the absolute errors in Ex. 5.1.

\begin{tabular}{|c|c|c|c|c|c|c|c|}
\hline $\mathrm{x}$ & Exact solution & MADM $_{4}(\mathrm{x})$ & $\left|\mathrm{E}_{4}\right|$ & MADM S $_{5}(\mathrm{x})$ & $\left|\mathrm{E}_{5}\right|$ & MADM $_{6}(\mathrm{x})$ & $\left|\mathrm{E}_{6}\right|$ \\
\hline 0.0 & 0.0 & 0.0 & 0.0 & 0.0 & 0.0 & 0.0 & 0.0 \\
\hline 0.2 & -0.06 & -0.0599999750 & 0.0000000249 & -0.0599999980 & 0.0000000019 & -0.0599999998 & 0.0000000001 \\
\hline 0.4 & -0.12 & -0.1199999531 & 0.0000000468 & -0.1199999964 & 0.0000000035 & -0.1199999997 & 0.0000000002 \\
\hline 0.6 & -0.18 & -0.1799999398 & 0.0000000601 & -0.1799999953 & 0.0000000046 & -0.1799999996 & 0.0000000003 \\
\hline 0.8 & -0.24 & -0.2399999373 & 0.0000000626 & -0.2399999951 & 0.0000000048 & -0.2399999996 & 0.0000000003 \\
\hline 1.0 & -0.30 & -0.2999999148 & 0.0000000851 & -0.2999999961 & 0.0000000038 & -0.2999999996 & 0.0000000003 \\
\hline
\end{tabular}

Table 3: Exact solution $g(x)=\frac{-3}{10} x$ and approximate solutions $S_{1}(x), S_{2}(x)$, and $S_{3}(x)$ utilizing the HAM along with the corresponding infinite norm of the absolute errors in Ex. 5.1.

\begin{tabular}{|c|c|c|c|c|c|c|c|}
\hline \multirow{2}{*}{$\mathrm{x}$} & \multirow{2}{*}{ Exact solution } & \multicolumn{2}{|c|}{$\hbar=-1.012033$} & \multicolumn{2}{c|}{$\hbar=-1.00463$} & \multicolumn{2}{c|}{$\hbar=-1.01064$} \\
\cline { 2 - 7 } & & $\mathrm{HAM}_{1}(\mathrm{x})$ & $\left|\mathrm{E}_{1}\right|$ & $\mathrm{HAM}_{2}(\mathrm{x})$ & $\left|\mathrm{E}_{2}\right|$ & $\mathrm{HAM} \mathrm{S}_{3}(\mathrm{x})$ & $\left|\mathrm{E}_{3}\right|$ \\
\hline 0.0 & 0.0 & 0.0 & 0.0 & 0.0 & 0.0 & 0.0 & 0.0 \\
\hline 0.2 & -0.06 & -0.0599287740 & 0.0000712259 & -0.0599956900 & 0.0000043099 & -0.0599997801 & 0.0000002198 \\
\hline 0.4 & -0.12 & -0.1198732907 & 0.0001267092 & -0.1199920201 & 0.0000079798 & -0.1199995857 & 0.0000004142 \\
\hline 0.6 & -0.18 & -0.1798740315 & 0.0001259684 & -0.1799897497 & 0.0000102502 & -0.1799994736 & 0.0000005263 \\
\hline 0.8 & -0.24 & -0.2399984652 & 0.0000015347 & -0.2399858406 & 0.0000141593 & -0.2399996226 & 0.0000003773 \\
\hline 1.0 & -0.30 & -0.3003410482 & 0.0003410482 & -0.2999634090 & 0.0000365909 & -0.3000013084 & 0.0000013084 \\
\hline
\end{tabular}

Table 4: Exact solution $g(x)=\frac{-3}{10} x$ and approximate solutions $S_{4}(x), S_{5}(x)$, and $S_{6}(x)$ utilizing the HAM along with the corresponding infinite norm of the absolute errors in Ex. 5.1.

\begin{tabular}{|c|c|c|c|c|c|c|c|}
\hline \multirow{2}{*}{$\mathrm{x}$} & \multirow{2}{*}{ Exact solution } & \multicolumn{2}{|c|}{$\hbar=-1.01115$} & \multicolumn{2}{c|}{$\hbar=-1.01561$} & \multicolumn{2}{c|}{$\hbar=-1.02019$} \\
\cline { 2 - 8 } & & $\mathrm{HAM}_{4}(\mathrm{x})$ & $\left|\mathrm{E}_{4}\right|$ & $\mathrm{HAM} \mathrm{S}_{5}(\mathrm{x})$ & $\left|\mathrm{E}_{5}\right|$ & $\mathrm{HAM} \mathrm{S}_{6}(\mathrm{x})$ & $\left|\mathrm{E}_{6}\right|$ \\
\hline 0.0 & 0.0 & 0.0 & 0.0 & 0.0 & 0.0 & 0.0 & 0.0 \\
\hline 0.2 & -0.06 & -0.0599999864 & 0.0000000135 & -0.0599999993 & 0.0000000006 & -0.0599999999 & 0.0000000000 \\
\hline 0.4 & -0.12 & -0.1199999745 & 0.0000000254 & -0.1199999986 & 0.0000000015 & -0.1199999998 & 0.0000000001 \\
\hline 0.6 & -0.18 & -0.1799999672 & 0.0000000327 & -0.1799999982 & 0.0000000017 & -0.1799999998 & 0.0000000001 \\
\hline 0.8 & -0.24 & -0.2399999610 & 0.0000000389 & -0.2399999984 & 0.0000000015 & -0.2399999997 & 0.0000000002 \\
\hline 1.0 & -0.30 & -0.2999998786 & 0.0000001213 & -0.3000000045 & 0.0000000045 & -0.2999999992 & 0.0000000007 \\
\hline
\end{tabular}


Table 5: Exact solution $g(x)=e^{-x}$ and approximate solutions $S_{1}(x), S_{2}(x)$, and $S_{3}(x)$ utilizing the MADM along with the corresponding infinite norm of the absolute errors in Ex. 5.2.

\begin{tabular}{|c|c|c|c|c|c|c|c|}
\hline $\mathrm{x}$ & Exact solution & ${\text { MADM } S_{1}(\mathrm{x})}$ & $\left|\mathrm{E}_{1}\right|$ & ${\text { MADM } \mathrm{S}_{2}(\mathrm{x})}$ & $\left|\mathrm{E}_{2}\right|$ & ${\text { MADM } \mathrm{S}_{3}(\mathrm{x})}$ & $\left|\mathrm{E}_{3}\right|$ \\
\hline 0.0 & 1.0 & 1.0 & 0.0 & 1.0 & 0.0 & 1.0 & 0.0 \\
\hline 0.2 & 0.8187307530 & 0.8187221386 & 0.0000086144 & 0.8187307607 & 0.0000000076 & 0.8187307538 & 0.0000000007 \\
\hline 0.4 & 0.6703200460 & 0.6700233694 & 0.0002966765 & 0.6703194053 & 0.0000006407 & 0.6703200481 & 0.0000000021 \\
\hline 0.6 & 0.5488116360 & 0.5465259146 & 0.0022857214 & 0.5487934433 & 0.0000181927 & 0.5488115461 & 0.0000000899 \\
\hline 0.8 & 0.4493289641 & 0.4396075519 & 0.0097214122 & 0.4491395263 & 0.0001894377 & 0.4493265280 & 0.0000024360 \\
\hline 1.0 & 0.3678794411 & 0.3379793924 & 0.0299000487 & 0.3667098048 & 0.0011696363 & 0.3678494169 & 0.0000300242 \\
\hline
\end{tabular}

Table 6: Exact solution $g(x)=e^{-x}$ and approximate solutions $S_{4}(x), S_{5}(x)$, and $S_{6}(x)$ utilizing the MADM along with the corresponding infinite norm of the absolute errors in Ex. 5.2.

\begin{tabular}{|c|c|c|c|c|c|c|c|}
\hline $\mathrm{x}$ & Exact solution & ${\text { MADM } S_{4}(\mathrm{x})}$ & $\left|\mathrm{E}_{4}\right|$ & ${\text { MADM } S_{5}(\mathrm{x})}$ & $\left|\mathrm{E}_{5}\right|$ & MADM $_{6}(\mathrm{x})$ & $\left|\mathrm{E}_{6}\right|$ \\
\hline 0.0 & 1.0 & 1.0 & 0.0 & 1.0 & 0.0 & 1.0 & 0.0 \\
\hline 0.2 & 0.8187307530 & 0.8187307537 & 0.0000000006 & 0.8187307537 & 0.0000000006 & 0.8187307537 & 0.0000000006 \\
\hline 0.4 & 0.6703200460 & 0.6703200485 & 0.0000000025 & 0.6703200485 & 0.0000000025 & 0.6703200485 & 0.0000000025 \\
\hline 0.6 & 0.5488116360 & 0.5488116414 & 0.0000000053 & 0.5488116418 & 0.0000000057 & 0.5488116418 & 0.0000000057 \\
\hline 0.8 & 0.4493289641 & 0.4493289510 & 0.0000000130 & 0.4493289743 & 0.0000000102 & 0.4493289745 & 0.0000000104 \\
\hline 1.0 & 0.3678794411 & 0.3678788848 & 0.0000005563 & 0.3678794494 & 0.0000000082 & 0.3678794580 & 0.0000000168 \\
\hline
\end{tabular}

Table 7: Exact solution $g(x)=e^{-x}$ and approximate solutions $S_{1}(x), S_{2}(x)$, and $S_{3}(x)$ utilizing the HAM along with the corresponding infinite norm of the absolute errors in Ex. 5.2.

\begin{tabular}{|c|c|c|c|c|c|c|c|}
\hline \multirow{2}{*}{$\mathrm{x}$} & \multirow{2}{*}{ Exact solution } & \multicolumn{2}{|c|}{$\hbar=-1.049902$} & \multicolumn{2}{c|}{$\hbar=-1.019211$} & \multicolumn{2}{c|}{$\hbar=-1.011092$} \\
\cline { 3 - 8 } & & $\mathrm{HAM}_{1}(\mathrm{x})$ & $\left|\mathrm{E}_{1}\right|$ & $\mathrm{HAM}_{2}(\mathrm{x})$ & $\left|\mathrm{E}_{2}\right|$ & $\mathrm{HAM} \mathrm{S}_{3}(\mathrm{x})$ & $\left|\mathrm{E}_{3}\right|$ \\
\hline 0.0 & 1.0 & 1.0 & 0.0 & 1.0 & 0.0 & 1.0 & 0.0 \\
\hline 0.2 & 0.8187307530 & 0.8196564066 & 0.0009256536 & 0.8187241857 & 0.0000065673 & 0.8187307759 & 0.0000000228 \\
\hline 0.4 & 0.6703200460 & 0.6735176601 & 0.0031976141 & 0.6703050462 & 0.0000149997 & 0.6703200552 & 0.0000000092 \\
\hline 0.6 & 0.5488116360 & 0.5538378184 & 0.0050261823 & 0.5488273261 & 0.0000156900 & 0.5488115120 & 0.0000001240 \\
\hline 0.8 & 0.4493289641 & 0.4515643949 & 0.0022354308 & 0.4494208494 & 0.0000918853 & 0.4493296027 & 0.0000006386 \\
\hline 1.0 & 0.3678794411 & 0.3548451653 & 0.0130342758 & 0.3676995429 & 0.0001798982 & 0.3678775384 & 0.0000019026 \\
\hline
\end{tabular}

Table 8: Exact solution $g(x)=e^{-x}$ and approximate solutions $S_{4}(x), S_{5}(x)$, and $S_{6}(x)$ utilizing the HAM along with the corresponding infinite norm of the absolute errors in Ex. 5.2.

\begin{tabular}{|c|c|c|c|c|c|c|c|}
\hline \multirow{2}{*}{$\mathrm{x}$} & \multirow{2}{*}{ Exact solution } & \multicolumn{2}{|c|}{$\hbar=-1.015997$} & \multicolumn{2}{c|}{$\hbar=-1.014091$} & \multicolumn{2}{c|}{$\hbar=-1.006894$} \\
\cline { 3 - 8 } & & $\mathrm{HAM}_{4}(\mathrm{x})$ & $\left|\mathrm{E}_{4}\right|$ & $\mathrm{HAM} \mathrm{S}_{5}(\mathrm{x})$ & $\left|\mathrm{E}_{5}\right|$ & $\mathrm{HAM}_{6}(\mathrm{x})$ & $\left|\mathrm{E}_{6}\right|$ \\
\hline 0.0 & 1.0 & 1.0 & 0.0 & 1.0 & 0.0 & 1.0 & 0.0 \\
\hline 0.2 & 0.8187307530 & 0.8187307526 & 0.0000000004 & 0.8187307537 & 0.0000000006 & 0.8187307537 & 0.0000000006 \\
\hline 0.4 & 0.6703200460 & 0.6703200479 & 0.0000000018 & 0.6703200485 & 0.0000000025 & 0.6703200485 & 0.0000000025 \\
\hline 0.6 & 0.5488116360 & 0.5488116473 & 0.0000000112 & 0.5488116418 & 0.0000000057 & 0.5488116418 & 0.0000000057 \\
\hline 0.8 & 0.4493289641 & 0.4493289588 & 0.00000000528 & 0.4493289747 & 0.0000000105 & 0.4493289745 & 0.0000000104 \\
\hline 1.0 & 0.3678794411 & 0.3678794827 & 0.0000000416 & 0.3678794572 & 0.0000000160 & 0.3678794581 & 0.0000000172 \\
\hline
\end{tabular}




\section{Conclusion}

In this work, we studied a general class of nonlinear integro-differential equations of order $n, n \in$ $\mathbb{Z}^{+} \backslash\{0\}$. We transformed this class of equations to an equivalent nonlinear Fredholm-Volterra integral equation (NF-VIE). We proved, under satisfying some sufficient conditions, the existence of continuous solutions for the corresponding NF-VIE and hence the existence of $n$-differentiable continuous solutions for the studied class of n-order integro-differential equations. The uniqueness of this solution is discussed as well. The modified Adomian's decomposition method (MADM) and the homotopy analysis method (HAM) are used to solve numerically the NF-VIE and study the convergence rate and error estimate of the approximate solution. The numerical results in this paper show that the two proposed techniques converge rapidly when we supposed the initial approximation $g_{0}(x)$ to be the same in both methods. But when we choose the convergence control parameter $\hbar$ such that the squared residual is minimized, the HAM may give an approximate solution that is more accurate and converges faster than the one obtained utilizing the MADM. For future research directions, we suggest studying the singular fractal model corresponding to Eq. (1.1), [15].

\section{Acknowledgements}

The authors thank the anonymous reviewers for their important and valuable comments.

\section{References}

[1] G. Adomian, Stochastic Systems, Academic Press, Orlando, (1983). 1

[2] G. Adomian, Nonlinear Stochastic Operator Equations, Academic Press, Orlando, (1986).

[3] G. Adomian, Solving Frontier Problems of Physics: The Decomposition Method, Kluwer Academic Publishers Group, Dordrecht, (1994). 1

[4] R. P. Agarwal, M. Meehan, D. O'Regan, Fixed Point Theory and Applications, Cambridge University Press, Cambridge, (2001). 2

[5] A. Alidema, A. Georgieva, Adomian decomposition method for solving two-dimensional nonlinear Volterra Fuzzy integral equations, AIP Conference Proceedings, 2018 (2018), 11 pages. 1

[6] H. O. Bakodah, M. Al-Mazmumy, S. O. Almuhalbedi, Solving system of integro differential equations using discrete Adomian decomposition method, J. Taibah Univ. Sci., 13 (2019), 805-812. 1

[7] M. M. Elborai, M. A. Abdou, M. I. Youssef, On Adomian's decomposition method for solving nonlocal perturbed stochastic fractional integro-differential equations, Life Sci. J., 10 (2013), 550-555. 1

[8] I. L. El-Kalla, Error analysis of Adomian series solution to a class of nonlinear differential equations, Appl. Math. E-Notes, 7 (2007), 214-221. 1

[9] A. Hamoud, A. Azeez, K. Ghadle, A study of some iterative methods for solving Fuzzy Volterra-Fredholm integral equations, Indones. J. Ele. Eng. Comput. Sci., 11 (2018), 1228-1235. 1

[10] A. A. Hamoud, K. P. Ghadle, The approximate solutions of fractional Volterra-Fredholm integro-differential equations by using analytical techniques, Probl. Anal. Issues Anal., 7 (2018), 41-58.

[11] A. Hamoud, K. Ghadle, Modified Adomian decomposition method for solving Fuzzy Volterra-Fredholm integral equations, J. Indian Math. Soc., 85 (2018), 53-69.

[12] A. A. Hamoud, K. P. Ghadle, Modified Laplace decomposition method for fractional Volterra-Fredholm integro-differential equations, J. Math. Model., 6 (2018), 91-104. 1

[13] A. A. Hamoud, K. P. Ghadle, Usage of the homotopy analysis method for solving fractional Volterra-Fredholm integrodifferential equation of the second kind, Tamkang J. Math., 49 (2018), 301-315. 1

[14] A. A. Hamoud, K. P. Ghadle, S. M. Atshan, The approximate solutions of fractional integro-differential equations by using modified Adomian decomposition method, Khayyam J. Math., 5 (2019), 21-39. 1

[15] J. H. He, A short review on analytical methods for to a fully fourth-order nonlinear integral boundary value problem with fractal derivatives, Inter. J. Numer. Methods Heat Fluid Flow, 30 (2020), 4933-4943. 8

[16] E. Hetmaniok, D. Slota, T. Trawinski, R. Witula, Usage of the homotopy analysis method for solving the nonlinear and linear integral equations of the second kind, Numer. Algorithms, 67 (2014), 163-185. 1

[17] M. H. Holmes, Introduction to Perturbation Methods, Springer, New York, (2013). 1

[18] M. Issa, A. Hamoud, K. Ghadle, Numerical solutions of Fuzzy integro-differential equations of the second kind, J. Math. Computer Sci., 23 (2021), 67-74. 1

[19] A. Kurt, O. Tasbozan, Approximate analytical solutions to conformable modified Burgers equation using homotopy analysis method, Ann. Math. Sil., 33 (2019), 159-167. 1 
[20] S. J. Liao, The proposed homotopy analysis technique for the solution of nonlinear problems, Ph.D. Thesis, Shanghai Jiao Tong University, (1992). 1

[21] S. J. Liao, Beyond Perturbation Introduction to the Homotopy Analysis Method, Chapman and Hall/CRC, U.S.A., (2003). 1, 4

[22] S. J. Liao, Homotopy Analysis Method in Nonlinear Differential Equation, Springer, Berlin, (2012). 1

[23] S. J. Liao, Advances In The Homotopy Analysis Method, World Scientific Publishing Co., Hackensack, (2014). 1

[24] S. Maitama, W. D. Zhao, Local fractional homotopy analysis method for solving non-differentiable problems on Cantor sets, Adv. Difference Equ., 2019 (2019), 22 pages. 1

[25] M. Marino, Instantons and large N: An Introduction to Non-Perturbative Methods in Quantum Field Theory, Cambridge University Press, Cambridge, (2015). 1

[26] T. Muta, Foundations of Quantum Chromodynamics: An Introduction to Perturbative Methods in Gauge Theories, Third ed., World Scientific Pub. Co., Hackensack, (2010). 1

[27] A. H. Nayfeh, Perturbation Methods, Wiley-Interscience, New York, (2000). 1

[28] J. Singh, D. Kumar, D. Baleanu, S. Rathore, An efficient numerical algorithm for the fractional drinfeld-sokolov-wilson equation, Appl. Math. Comput., 335 (2018), 12-24. 1

[29] R. Singh, G. Nelakanti, J. Kumar, Approximate solution of Urysohn integral equations using the Adomian decomposition method, Sci. World J., 2014 (2014), 6 pages. 1

[30] A. M. Wazwaz, A reliable modification of Adomian decomposition method, Appl. Math. Comput., 102 (1999), 77-86. 3

[31] L.-J. Xie, A new modification of Adomian decomposition method for Volterra integral equations of the second kind, J. Appl. Math., 2013 (2013), 7 pages. 1 